\title{
CULTURA DE SEGURANÇA ENTRE PROFISSIONAIS DE SAÚDE EM HOSPITAL DE ENSINO
}

\author{
Elaine Drehmer de Almeida Cruz ${ }^{1}$, Denise Jorge Munhoz da Rocha ${ }^{2}$, Aline Batista Mauricio ${ }^{3}$, Francielle de \\ Souza Ulbrich ${ }^{3}$, Josemar Batista ${ }^{4}$, Eliane Cristina Sanches Maziero ${ }^{5}$
}

\begin{abstract}
RESUMO: Objetivo: avaliar a cultura de segurança organizacional entre os profissionais de um hospital de Ensino. Método: estudo tipo survey com 645 profissionais de um hospital de ensino no Estado do Paraná, de outubro de 2014 a julho de 2015, utilizando instrumento autoaplicável da Agency for Health care Research and Quality. Variáveis quantitativas foram representadas em percentuais; quando $\geq 75 \%$ das respostas foram positivas, cultura de segurança satisfatória; 74 a $51 \%$ neutra; e $\leq 50 \%$ frágil. A confiabilidade foi mensurada pelo coeficiente Alfa de Cronbach. Resultados: maior índice foi obtido na dimensão "Expectativas sobre o seu supervisor/chefe e ações promotoras da segurança", com 70,8\% $(n=455)$ de respostas positivas e "Respostas não punitivas ao erro" o menor, com 25,6\% $(n=164)$. O coeficiente Alfa de Cronbach obteve média de 0,62 , mostrando baixa confiabilidade. Conclusão: resultados mostram cultura de segurança insatisfatória e relevância de ações de promoção, com destaque à abordagem coletiva e não punitiva de erros.
\end{abstract}

DESCRITORES: Cultura organizacional; Pessoal de saúde; Segurança do paciente; Gestão da qualidade; Indicadores de qualidade em assistência à saúde.

\section{SAFETY CULTURE AMONG HEALTH PROFESSIONALS IN A TEACHING HOSPITAL}

\begin{abstract}
Objective: assess the safety culture among health professionals of a teaching hospital. Method: Cross-sectional surveytype study with 645 professionals of a teaching hospital of the State of Paraná, from October 2014 to July 2015, through the use of a self-administered instrument of the Agency for Health care Research and Quality. Quantitative variables were represented in percentage values: the safety culture was considered satisfactory if $\geq 75 \%$ of the responses were positive; neutral, if 74 to $51 \%$ of the responses were positive, and fragile, if $\leq 50 \%$ of the responses were positive. Reliability was measured by Cronbach's alpha coefficient. Results: the highest value was obtained for dimension "Expectations about your supervisor/boss and safety-promoting actions", with $70.8 \%$ ( $n=455)$ of positive responses and "Non punitive responses to error" obtained the lowest value, with $25.6 \%$ ( $n=164)$. The Cronbach's alpha coefficient obtained an average of 0.62 , showing low reliability Conclusion: The results reveal an unsatisfactory safety culture and stress the importance of actions targeted to the promotion of a safety culture, with emphasis to a collective and non-punitive approach to errors.
\end{abstract}

DESCRIPTORS: Organizational culture; Health personnel; Patient safety; Quality management; Indicators of healthcare quality.

\section{CULTURA DE SEGURIDAD ENTRE PROFESIONALES DE SALUD EN HOSPITAL DE ENSEÑANZA}

RESUMEN:Objetivo: Evaluar la cultura de seguridad organizacional entre los profesionales de un hospital de enseñanza. Método: Estudio tipo survey con 645 profesionales de un hospital de enseñanza en Estado de Paraná, de octubre de 2014 a julio de 2015 , con instrumento auto aplicable de Agency for Health care Research and Quality. Variables cuantitativas fueron representadas en porcentajes; cuando $\geq 75 \%$ de las respuestas fueron positivas, cultura de seguridad satisfactoria; 74 a $51 \%$ neutra; y $\leq 50 \%$ débil. La confiabilidad fue mensurada por el coeficiente Alfa de Cronbach. Resultado: Mayor índice fue obtenido en la dimensión "Expectativas sobre el supervisor/jefe y acciones promotoras da seguridad", con $70,8 \%$ ( $n=455)$ de respuestas positivas y "Respuestas no punitivas al error" el menor, con 25,6\% ( $n=164)$. El coeficiente Alfa de Cronbach obtuvo media de 0,62, presentando baja confiabilidad. Conclusión: Los resultados muestran cultura de seguridad insatisfactoria y relevancia de acciones de promoción, destacándose abordaje colectivo y no punitivo de errores.

DESCRIPTORES: Cultura organizacional; Personal de salud; Seguridad del paciente; Gestión de la cualidad; Indicadores de cualidad en asistencia a la salud.

\footnotetext{
'Enfermeira. Doutora em Enfermagem. Docente de Enfermagem da Universidade Federal do Paraná. Curitiba, PR, Brasil. Hospital de Clínicas - Universidade Federal do Paraná. Curitiba, PR, Brasil.

${ }^{3}$ Discente de Enfermagem. Universidade Federal do Paraná. Curitiba, PR, Brasil.

${ }^{4}$ Enfermeiro. Mestrando em Enfermagem. Universidade Federal do Paraná. Curitiba, PR, Brasil.

${ }^{5}$ Enfermeira. Doutoranda em Enfermagem. Universidade Federal do Paraná. Curitiba, PR, Brasil.
} ${ }^{2}$ Enfermeira. Mestre em Enfermagem. Chefe do Serviço de Vigilância em Saúde e Segurança do Paciente do Complexo do

\section{Autor Correspondente:}

Eliane Cristina Sanches Maziero

Universidade Federal do Paraná

R. Santa Ana, 53, - 83.405-070 - Colombo, PR, Brasil

E-mail: elicris_maziero@yahoo.com.br
Recebido: 17/02/2017

Finalizado: 16/11/2017 


\section{INTRODUÇÃO}

A redução, a um mínimo aceitável, do risco de dano desnecessário associado ao cuidado de saúde compõe o conceito de segurança do paciente da Organização Mundial da Saúde ${ }^{(1)}$ e, portanto, pressupõe que na assistência em saúde o erro zero é improvável. Embora inerente à natureza do trabalho, incidentes e eventos adversos são evitáveis e multifatoriais; melhores condições de trabalho relativas à estrutura, organização, qualificação e força de trabalho contribuem para a prevenção de danos.

Neste contexto, o conjunto de valores, compromisso e competência de uma organização de saúde contribui para a promoção da segurança do paciente e representa a cultura de segurança institucional ${ }^{(1)}$. Essa influencia diretamente na qualidade da assistência prestada, por meio de atitudes dos profissionais e líderes, com vistas a buscar medidas e soluções que minimizem riscos e danos evitáveis ${ }^{(2)}$.

O conceito cultura de segurança surgiu na indústria, após o desastre nuclear em Chernobyl, e passou a regulamentar e investigar fatores organizacionais, e não apenas individuais; da mesma forma, seu uso na área da saúde reitera a importância da visão global da organização de saúde, e não ações individuais, na ocorrência de erros ${ }^{(3)}$. Deste modo, a cultura de segurança organizacional consiste em um conjunto de crenças partilhadas que sustentam práticas seguras entre os profissionais que nela trabalham. Tal cultura é marcada pela comunicação aberta, trabalho em equipe, reconhecimento da dependência mútua e a primazia da segurança como uma prioridade em todos os níveis da organização ${ }^{(1)}$.

Para alcançar a assistência qualificada e segura, é necessário que a cultura de segurança esteja em pauta nas instituições, de maneira que a gerência incentive os profissionais a promover essa segurança ${ }^{(3)}$. Com o intuito de sua avaliação, foi criado em 2004 o instrumento Hospital Surveyon Patient Safety Culture (HSOPSC), pela Agency for Health care Research and Quality (AHRQ). No Brasil, esse foi traduzido e adaptado transculturalmente por $\operatorname{Reis}^{(4)}$ e é composto por 12 dimensões da cultura de segurança, as quais permitem conhecer potencialidades e fragilidades, e contribuir para o planejamento de ações com vistas a promover a segurança da assistência prestada em serviços de saúde hospitalar.

Reconhecendo a importância e atualidade do tema, o objetivo desta pesquisa foi avaliar a cultura de segurança organizacional de um hospital de ensino do Estado do Paraná.

\section{MÉTODO}

Trata-se de pesquisa avaliativa de abordagem quantitativa do tipo survey transversal. O local do estudo foi composto pelos serviços e unidades de assistência à saúde de um hospital de ensino de grande porte de Curitiba-PR; os dados foram coletados entre outubro de 2014 e julho de 2015, utilizando o questionário HSOPSC traduzido e validado para o Brasil ${ }^{(4)}$.

O questionário HSOPSC é autoaplicável e contém 50 itens, 44 especificamente sobre cultura de segurança e seis relacionados a informações pessoais. Das 12 dimensões da cultura de segurança organizacional, três são relacionadas ao hospital, sete à unidade de trabalho dentro do hospital e duas constituem variáveis de resultados, relativas ao julgamento do grau de segurança atribuído e ao número de eventos notificados pelo respondente. Os itens, em sua maioria, são respondidos utilizando uma escala de concordância ou de frequência de cinco pontos ${ }^{(5)}$.

Foram consideradas respostas positivas aquelas cujos participantes concordavam/concordavam totalmente para perguntas formuladas positivamente; aquelas formuladas negativamente tiveram as respostas invertidas para a apresentação dos resultados e análise. Quando $\geq 75 \%$ de respostas positivas, a dimensão é considerada forte para a segurança do paciente, e resultados $\leq 50 \%$ indicam fragilidade e necessidade de melhorias na segurança do paciente ${ }^{(5)}$. Respostas neutras são aquelas cujo percentual de resposta varia entre 51 e $74 \%$.

A avaliação da confiabilidade do HSOPSC se deu por meio da análise da consistência interna das dimensões, através do cálculo do coeficiente Alfa de Cronbach. Este é comumente utilizado para 
mensurar a consistência interna e é adequado para situações em que se aplica o instrumento uma única vez em determinada amostra. Os valores variam entre 0 e 1 e quanto mais o valor se aproximar de um, mais consistente e, consequentemente, mais confiável o instrumento ${ }^{(4)}$.

A amostragem da pesquisa foi não probabilística, a população alvo foi constituída por 1.807 profissionais e trabalhadores lotados no hospital, com carga horária semanal mínima de 20 horas e atividade predominantemente assistencial e/ou com atendimento ao paciente. Foram excluídos os participantes que responderam menos de $50 \%$ das perguntas e/ou com pelo menos uma seção completa não preenchida ${ }^{(5)}$. Após ampla divulgação da pesquisa nos meios de comunicação do hospital, a população alvo foi abordada diretamente em seu ambiente de trabalho, individualmente e/ou em grupos, para o convite e informações adicionais sobre a pesquisa. Também foram disponibilizados locais específicos para os esclarecimentos e coleta de dados. Foram respeitados preceitos de ética em pesquisa com seres humanos e este estudo obteve aprovação do Comitê de Ética institucional sob protocolo CEP/SD 241.958.

Um banco de dados elaborado no programa Microsoft Office Exce $^{\circledR}$ foi alimentado por dupla digitação, seguida de verificação e correção de inconsistências. Os resultados das variáveis quantitativas foram descritos por média, desvio padrão, mediana, valor mínimo e valor máximo; as variáveis qualitativas foram apresentadas em frequências e percentuais. Para cada uma das dimensões, foi estimado o coeficiente Alfa de Cronbach e os dados foram analisados com auxílio do programa computacional IBM SPPS Statistics v.20 e assessoria de profissional estatístico.

\section{RESULTADOS}

Foram respondidos 744 instrumentos, representando $41,17 \%$ da população-alvo. Aplicando-se os critérios da pesquisa, 99 foram excluídos, a amostra final de participantes representou 35,69\% ( $n=645)$ da população alvo.

Os resultados apresentados referem-se aos itens respondidos pelos participantes, caracterizados em $88 \%(n=568)$ pelo sexo feminino, $22 \%(n=142)$ com curso superior completo e $34,2 \%(n=221)$ com especialização. A idade média variou entre 33,9 e 49,9 anos, com mediana entre 26 e 50 anos com desvio padrão entre 6,9 e 12,3. Entre os respondentes $29,5 \%(n=190)$ tinham entre 11 e 20 anos de atuação no hospital e 31,3\% ( $n=202)$ trabalhavam há mais de 20 anos na instituição. Porém, $21,5 \%(n=139)$ estavam lotados na unidade de trabalho há menos de um ano e $27,9 \%(n=180)$ entre um e cinco anos.

Quanto à carga horária semanal, 58,3\% $(n=376)$ referiram trabalhar de 20 a 39 horas semanais; $81,8 \%(n=528)$ não trabalhavam em outra instituição e entre os que possuíam dois vínculos $40 \%$ ( $n=$ 48) referiram carga adicional inferior a 20 horas e $48,7 \%(n=57)$ entre 20 e 39 horas a mais por semana. Quanto ao turno, 34,4\% $(n=222)$ dos participantes referiu trabalhar em turno integral, 16,3\% $(n=105)$ à tarde e $12,2 \%(n=79)$ no período noturno.

Em relação ao cargo ou função, os participantes atuavam, em média, há 16 anos na função, 89,5\% $(n=566)$ exerciam atividade essencialmente assistencial e $84,6 \%(n=535)$ tinha contato direto com os pacientes; majoritariamente a amostra representa a enfermagem (Tabela 1).

Tabela 1 - Cargo dos participantes da pesquisa cultura de segurança organizacional. Curitiba, PR, Brasil, 2015 (continua)

\begin{tabular}{|c|c|c|c|c|c|}
\hline \multirow[t]{2}{*}{ Cargo/função } & \multicolumn{2}{|c|}{ População-alvo } & \multicolumn{2}{|c|}{ Participantes } & \multirow{2}{*}{$\begin{array}{c}\begin{array}{c}\text { Relação Participantes } \\
\text { e População alvo }\end{array} \\
\%\end{array}$} \\
\hline & $\bar{n}$ & $\%$ & $\mathbf{n}$ & $\%$ & \\
\hline Auxiliar de enfermagem & 485 & 26,8 & 176 & 27,8 & 32,3 \\
\hline Enfermeiro & 240 & 13,3 & 108 & 17,1 & 45 \\
\hline Técnico de enfermagem & 189 & 10,5 & 72 & 11,4 & 38,1 \\
\hline Médico & 332 & 18,4 & 32 & 5,1 & 9,6 \\
\hline Médico residente/em treinamento & 320 & 17,7 & 30 & 4,7 & 9,4 \\
\hline
\end{tabular}




\begin{tabular}{lccccc}
\hline Outros nível superior* & 128 & 7,1 & 106 & 16,8 & 82,8 \\
\hline Outros nível médio** & 113 & 6,2 & 108 & 17,1 & 95,6 \\
\hline Total & 1807 & 100 & 632 & 100 & 35
\end{tabular}

Nota: 13 participantes não responderam

*Assistente social; Terapeuta ocupacional; Psicólogo, Odontólogo, Nutricionista, Fonoaudiólogo, Fisioterapeuta, Farmacêutico.

** Técnico de Laboratório, Atendente de enfermagem, Auxiliar de nutrição, Auxiliar administrativo, Instrumentador cirúrgico.

A Tabela 2 apresenta os resultados da cultura de segurança organizacional em cada uma das 12 dimensões; nenhuma foi considerada forte ( $\geq 75 \%)$. Quatro foram consideradas neutras (51 a $74 \%$ ), com destaque para "Expectativas sobre o seu supervisor/chefe e ações promotoras da segurança" e "Trabalho em equipe dentro das unidades"; e oito dimensões foram consideradas frágeis $(\leq 50 \%)$, sendo a mais crítica "Respostas não punitivas ao erro".

Tabela 2 - Dimensões da cultura de segurança organizacional (N=642). Curitiba, PR, Brasil, 2015

\begin{tabular}{|c|c|c|c|}
\hline \multirow[t]{2}{*}{ Dimensão } & \multicolumn{3}{|c|}{ Percentual médio } \\
\hline & $\begin{array}{l}\text { Resposta } \\
\text { Negativa }\end{array}$ & $\begin{array}{l}\text { Resposta } \\
\text { Neutra }\end{array}$ & $\begin{array}{l}\text { Resposta } \\
\text { Positiva }\end{array}$ \\
\hline 1.Trabalho em equipe dentro das unidades & 21,9 & 10,1 & 68 \\
\hline $\begin{array}{l}\text { 2.Expectativas sobre seu supervisor/chefe e ações } \\
\text { promotoras da segurança }\end{array}$ & 13,9 & 15,4 & 70,8 \\
\hline 3.Aprendizado organizacional - melhoria continua & 18 & 15,9 & 66,1 \\
\hline 4.Apoio da gestão para segurança do paciente & 35,8 & 25,1 & 39,1 \\
\hline 5.Percepção geral da segurança do paciente & 40,9 & 12,4 & 46,7 \\
\hline 6.Retorno da informação e comunicação sobre o erro & 24,4 & 29,6 & 46,1 \\
\hline 7.Abertura da comunicação & 18,9 & 25,9 & 55,2 \\
\hline 8.Frequência de relatos de eventos & 28,6 & 22,2 & 49,2 \\
\hline 9.Trabalho em equipe entre unidades & 40,2 & 25 & 34,8 \\
\hline 10.Adequação de profissionais & 47,9 & 14,9 & 37,1 \\
\hline 11.Passagem de plantão/turno e transferências & 43,4 & 22 & 34,6 \\
\hline 12.Respostas não punitivas ao erro & 58 & 16,4 & 25,6 \\
\hline
\end{tabular}

Quanto às respostas dadas para as questões em cada dimensão, destacamos como resultados positivos as relativas às perguntas "Estamos ativamente fazendo coisas para melhorar a segurança do paciente" com 82,9\% ( $n=532)$ e "O meu chefe/supervisor não dá atenção aos problemas de segurança do paciente que acontecem repetidamente", com 81,6\% $(n=524)$. Estas perguntas são referentes às dimensões um e dois (Trabalho em equipe dentro das unidades e Expectativas sobre seu supervisor/ chefe e ações promotoras da segurança), respectivamente.

Resultados negativos surgiram referentes às perguntas "A segurança do paciente jamais é comprometida em função de maior quantidade de trabalho a ser concluída", apontada por 32,8\% ( $n=211)$, "Nesta unidade temos problemas de segurança do paciente" por 38,2\% (n=245), ambas da dimensão nove (Trabalho em equipe dentro das unidades) e "As unidades do hospital não estão coordenadas entre si" por 26,2\% ( $n=168$ ), dimensão seis (Retorno da informação e comunicação sobre o erro).

No entanto, as questões que obtiveram o maior índice de respostas negativas foram "Temos profissionais suficientes para dar conta da carga de trabalho" com 72,6\% $(n=466)$ e "Os profissionais consideram que seus erros podem ser usados contra eles" com 63,6\% ( $n=408)$ de respondentes, sendo ambas as questões pertencentes à dimensão nove (Trabalho em equipe dentro das unidades). 
A nota atribuída pelos participantes à segurança do paciente foi, respectivamente, Muito boa (48,9\%), Regular (38,5\%), Excelente (7,3\%), Ruim (4,2) e Muito Ruim (1,1\%). Quanto à notificação de adversos nos 12 meses anteriores à pesquisa, 365 (56,6\%) responderam nunca ter notificado; o enfermeiro foi quem mais notificou entre médicos e equipe de enfermagem (Tabela 3 ).

Tabela 3 - Número de eventos adversos notificados segundo a categoria profissional. Curitiba, PR, Brasil, 2015

\begin{tabular}{lcccccc}
$\begin{array}{l}N^{*} \text { de } \\
\text { eventos }\end{array}$ & Geral & Enfermeiro & $\begin{array}{c}\text { Técnico por categoria profissional } \\
\text { Enfermagem }\end{array}$ & $\begin{array}{c}\text { Auxiliar } \\
\text { Enfermagem }\end{array}$ & Médico & $\begin{array}{c}\text { Médico } \\
\text { residente/em } \\
\text { treinamento }\end{array}$ \\
\hline Nenhum & 56,6 & 20,4 & 51,4 & 70,5 & 56,3 & 46,7 \\
\hline $1-2$ & 20,1 & 13,9 & 33,3 & 18,8 & 31,3 & 36,7 \\
\hline $3-5$ & 11,9 & 31,5 & 8,3 & 6,3 & - & 13,3 \\
\hline $6-10$ & 6,9 & 20,4 & 4,2 & 3,4 & 3,1 & 3,3 \\
\hline $11-20$ & 2,3 & 8,3 & - & 0,6 & 3,1 & - \\
\hline$\geq 21$ & 2,2 & 5,6 & 2,8 & 0,6 & 6,3 & -
\end{tabular}

A confiabilidade do instrumento, mensurado pelo coeficiente Alfa de Cronbach, obteve média de 0,62. As dimensões "Respostas não punitivas ao erro" e "Percepção geral da segurança do paciente" foram as que obtiveram o menor valor do coeficiente, com 0,47. As dimensões "Frequência de relatos de eventos" e "Apoio da gestão para segurança do paciente" obtiveram os maiores valores do coeficiente com 0,81 e 0,77 , respectivamente (Tabela 4 ).

Tabela 4 - Valores do coeficiente Alfa de Cronbach para cada dimensão da cultura de segurança organizacional. Curitiba, PR, Brasil, 2015

\section{Dimensão}

$\mathbf{N}^{\circ}$ de questões Alfa de Cronbach

\begin{tabular}{lll}
\hline Trabalho em equipe dentro das unidades & 4 & 0,63 \\
\hline Expectativas sobre o supervisor e ações promotoras da segurança & 4 & 0,67 \\
\hline Aprendizado organizacional - melhoria contínua & 3 & 0,49 \\
\hline Apoio da gestão para segurança do paciente & 3 & 0,77 \\
\hline Percepção geral da segurança do paciente & 4 & 0,47 \\
\hline Retorno da informação e comunicação sobre o erro & 3 & 0,68 \\
\hline Abertura da comunicação & 3 & 0,67 \\
\hline Frequência de relatos de eventos & 3 & 0,81 \\
\hline Trabalho em equipe entre unidades & 4 & 0,59 \\
\hline Adequação de profissionais & 4 & 0,49 \\
\hline Passagem de plantão/turno e transferências & 3 & 0,66 \\
\hline Respostas não punitivas ao erro & 3 & 0,47
\end{tabular}

\section{DISCUSSÃO}

Os resultados obtidos com a aplicação do questionário nos permitem a avaliação da cultura de segurança em diversos aspectos, embora nenhuma dimensão tenha apresentado resultado satisfatório, evidenciando áreas frágeis de cultura de segurança nas 12 dimensões. Quanto à confiabilidade do 
instrumento, ressalta-se a baixa consistência interna na maioria das dimensões investigadas.

Esses resultados são preocupantes ao considerar a relação entre baixos escores de cultura de segurança e ocorrência de eventos adversos ${ }^{(6)}$ bem como a consistência interna evidenciada no uso deste instrumento, na instituição da pesquisa.

Quando comparado a um estudo realizado em três unidades de terapia intensiva utilizando o mesmo instrumento, encontra-se semelhança. Na dimensão dois (Expectativas sobre seu supervisor/ chefe e ações promotoras da segurança do paciente) obteve-se $75,4 \%$ de respostas positivas ${ }^{(7)}$. Já o relatório da $\mathrm{AHRQ}{ }^{(8)}$, relativo a dados de 600 hospitais norte-americanos, demonstrou similaridade, com $78 \%$ de respostas positivas.

A presente pesquisa demonstra que essa é uma área fortalecida para a cultura de segurança na instituição $(70,8 \%)$, indicando que líderes consideram as sugestões e opiniões da equipe na promoção da segurança do paciente e incentivam os funcionários a aderir às normas de segurança, aprender e promover mudanças com os erros que são comunicados. Isso pode ser demonstrado por meio da afirmação "O meu supervisor/chefe não dá atenção aos problemas de segurança do paciente que acontecem repetidamente" na qual $81,6 \%$ dos profissionais responderam que "discordam" ou "discordam totalmente".

A questão "Estamos ativamente fazendo coisas para melhorar a segurança do paciente", pertencente à dimensão três (Aprendizado organizacional - melhoria contínua), obteve a maior porcentagem de respostas positivas (82,9\%). Isso reforça que, apesar da escassez de profissionais e uma cultura organizacional punitiva frente aos erros, os profissionais demonstram preocupação quanto à segurança do paciente, por meio do desenvolvimento de ações constantes e apontamento de situações que possam afetar negativamente o cuidado.

Na dimensão 12 (Respostas não punitivas ao erro), semelhantemente, houve menor porcentagem de respostas positivas em estudo paulista ${ }^{(7)}$ assim como nos hospitais norte-americanos ${ }^{(8)}$ representando $29,6 \%$ e $45 \%$, respectivamente, quando comparada aos resultados desta pesquisa ( $25,6 \%)$. Por outra via, percebemos que a cultura punitiva frente aos erros ainda é um fator de fragilidade nas instituições de saúde, não somente no Brasil. Ainda, há persistência da cultura de culpabilização dos indivíduos; erros são vistos como consequências de fatores pessoais e decorrentes da falta de competência ou atenção. Porém, esses acontecem por meio de um conjunto de falhas sequenciais existentes no sistema de saúde, e não meramente por um fator isolado, como o descuido do profissional ${ }^{(9)}$.

Estudos evidenciam que erros decorrentes de um conjunto de falhas não devem ser tratados de maneira isolada. Portanto, para que a resposta não punitiva ao erro seja identificada comoárea fortalecida em cultura de segurança, é necessário evidenciar as falhas do sistema, sem punir isoladamente ${ }^{(10)}$. A abordagem predominantemente individual impede que sejam realizadas mudanças em busca de progressos globais na organização. Uma cultura punitiva pode prejudicar o reconhecimento do erro pelos profissionais de saúde, desencorajar a notificação e, assim, impedir o aprendizado organizacional a partir da sua ocorrência ${ }^{(10)}$.

Um exemplo disso é o alto percentual de profissionais que, neste estudo, afirmaram não ter notificado nenhum evento nos últimos 12 meses ou de um a cinco eventos (88,6\%), demonstrando uma baixa adesão ao sistema de notificação institucional. Resultados esses que corroboram com estudo baiano, no qual aproximadamente $88 \%$ dos profissionais informaram não terem notificado eventos ${ }^{(11)}$.

Outro dado que reforça essa questão foi a afirmativa "Os profissionais consideram que seus erros podem ser usados contra eles", pertencente à dimensão 12 (Respostas não punitivas ao erro), na qual $63,6 \%$ "concordam" ou "concordam totalmente", demonstrando que se sentem intimidados quanto à comunicação de seus erros. Pesquisa conduzida no sudeste do Brasil verificou que 72,5\% dos profissionais afirmaram que seus erros podem ser utilizados contra eles ${ }^{(12)}$. Esses achados reforçam outras investigações brasileiras que apontam a dificuldade de discussão entre os trabalhadores de saúde referente aos erros decorrentes do cuidado ${ }^{(13-14)}$.

A dimensão um (Trabalho em equipe entre unidades) apresentou a maior porcentagem de respostas positivas $(82 \%)$ no relatório norte-americano ${ }^{(8)}$, e no presente trabalho obteve $68 \%$ de respostas positivas. Essa dimensão apresentou escore semelhante a outros estudos brasileiros que identificaram 
valores inferiores ao mínimo recomendado para se considerar como área fortalecida para a segurança do paciente $\mathrm{e}^{(11,13-14)}$.

Outro fator a destacar é a sobrecarga de trabalho referida devido à escassez de profissionais. "Temos profissionais suficientes para dar conta da carga de trabalho", pertencente à dimensão 10 (Adequação de profissionais), foi a questão que obteve o maior índice de respostas negativas, com $72,6 \%$. Percentual esse elevado devido à resposta dos profissionais de Enfermagem, os quais são a maior força de trabalho em instituições hospitalares. Uma pesquisa realizada em duas unidades de terapia intensiva de hospitais públicos brasileiros detectou que, aproximadamente, $78 \%$ dos incidentes sem lesão e de eventos adversos em pacientes foram relacionados à assistência da Enfermagem, sendo atribuídos à sobrecarga de trabalho e aumento de risco de mortalidade dos pacientes ${ }^{(15)}$.

Quanto ao objetivo de cada dimensão, salientamos que a dimensão quatro (Apoio da gestão para segurança do paciente) avalia se a gestão hospitalar promove segurança ao paciente e aborda o tema como prioridade nas unidades; a dimensão um (Trabalho em equipe entre as unidades) avalia se as unidades do hospital cooperam entre si para assegurar a melhor qualidade na assistência; a 10 (Adequação de profissionais) avalia se a carga horária dos funcionários está adequada ao planejamento do cuidado dos pacientes; a dimensão 11 (Passagem de plantão/turno e transferências) avalia se as informações importantes para a continuidade do cuidado são transmitidas nas passagens de plantão; e a 12 (Resposta não punitiva ao erro) avalia como os funcionários se sentem em relação aos seus erros ${ }^{(4)}$.

A partir dos resultados desta pesquisa, nota-se que os colaboradores da instituição percebem que a gestão não promove um clima de trabalho adequado para a segurança do paciente, mostramse insatisfeitos com a carga de trabalho e percebem que seus erros poderiam gerar punições. Essas constatações vão ao encontro a outro estudo realizado sobre cultura de segurança do paciente ${ }^{(16)}$.

Em relaçãoà dimensão de apoio da gestão, os resultados indicam a necessidade de maior envolvimento dessa para a promoção da segurança do paciente, por meio do incentivo e comprometimento das lideranças, com possibilidade de aumento dos indicadores de qualidade das instituições. Mediante a verificação das áreas frágeis em cultura de segurança, é essencial o envolvimento e comprometimento de toda a instituição e, principalmente, da gestão hospitalar, que tem o papel de demonstrar interesse na promoção de segurança do paciente, garantir a melhoria da assistência entre as equipes de saúde, e instituir a comunicação aberta sobre $\operatorname{erros}^{(17)}$.

O conhecimento e habilidades, juntamente com condições de trabalho como supervisão, comunicação e equipamentos, são relevantes à segurança. Essas variáveis são afetadas pelos processos organizacionais e decisões gerenciais, impactando sobre a segurança assistencial ${ }^{(7)}$.

Em relação à formação profissional dos participantes deste estudo, percebe-se que esses buscam se especializar, o que é um ponto positivo quando se trata de segurança do paciente; pesquisadores defendem que a pós-graduação permite ao profissional consenso entre seu exercício e seu discurso. A qualificação também contribui para a mudança de atitude e da postura do profissional frente à assistência de qualidade, promove a responsabilidade com a instituição e a busca contínua de melhores condições para sua prática(18)

Também foi possível constatar que $89,5 \%$ dos participantes exerciam atividade essencialmente assistencial e interação ou contato direto com o paciente. Embora a cultura de segurança do paciente deva permear todas as esferas da organização(19), aqueles profissionais que atuam diretamente na assistência certamente irão interferir mais constantemente na segurança do atendimento, o que resulta na necessidade de serem continuamente estimulados para a promoção da própria cultura de segurança ${ }^{(20)}$.

Nesse contexto, destaca-se a importância da cultura de segurança na instituição com a segurança do paciente atendido, associação estudada em diversos países. Estudo realizado em unidades de urgência e emergência da Holanda identificou dimensões positivas da cultura associadas com a segurança do paciente ${ }^{(21)}$. Outro, realizado em 68 hospitais libaneses, com 6.807 profissionais de saúde, encontrou indícios significativos da relação da cultura positiva com a segurança do paciente ${ }^{(22)}$. Pesquisa conduzida em sete hospitais da China confirmou a hipótese de que uma cultura positiva está relacionada à minimização na ocorrência de eventos adversos ${ }^{(23)}$. 
Autores defendem que mudanças na cultura organizacional e na cultura individual do profissional de saúde possam influenciar positivamente na cultura de segurança do paciente, tornando-se essencial para a assistência segura e de qualidade, já que impactam na motivação e comportamentos seguros, com reflexos na prática diária ${ }^{(24)}$.

Entre as limitações do estudo, aponta-se a baixa adesão ao preenchimento do questionário por determinadas categorias profissionais e a ausência de análise estatística inferencial, podendo ser realizada em estudos subsequentes.

\section{CONCLUSÃO}

A amostra desta pesquisa, constituída majoritariamente por trabalhadores e profissionais experientes e maduros que interagem e prestam assistência aos pacientes, chancela os resultados obtidos, os quais mostram que na cultura de segurança organizacional do hospital há importantes fragilidades relativas à comunicação, trabalho em equipe, adequação de profissionais, apoio da gestão e, principalmente, percepção do risco de punição frente ao erro e baixa notificação de eventos adversos.

Paradoxalmente, os participantes consideram a segurança do paciente na instituição como muito boa e reconhecem seu papel ativo, e dos supervisores, na construção desse importante indicador da qualidade assistencial.

Apesar da baixa consistência interna do instrumento, conclui-se haver pontos críticos para a segurança do paciente que podem subsidiar o planejamento de ações gerenciais sistêmicas com vistas a promover a cultura de segurança organizacional, a partir das lacunas identificadas e detalhadas de acordo com as diferentes dimensões.

\section{REFERÊNCIAS}

1. Organização Mundial da Saúde (OMS). The Conceptual Framework for the International Classification for Patient Safety: Final Technical Report. [Internet] Geneva: WHO; 2009 [acesso em 21 jul 2016]. Disponível: http:// www.who.int/patientsafety/taxonomy/icps_full_report.pdf.

2. Barros AA, Oliveira RM, Pinheiro AC, Leitão IMTA, do Vale AP, da Silva LMS. Práticas de incentivo à cultura de segurança por lideranças de enfermagem segundo enfermeiros assistenciais. Rev enferm UFPE online. [Internet] 2014;8(12) [acesso em 09 jul 2016]. Disponível: http://www.revista.ufpe.br/revistaenfermagem/index.php/revista/ article/viewFile/6753/pdf_6765.

3. Calori MAO, Gutierrez SL, Guidi TAC. Segurança do paciente: Promovendo a cultura de segurança. Saúde em Foco. [Internet] 2015;(7) [acesso em 10 jul 2016]. Disponível: http://www.unifia.edu.br/revista_eletronica/revistas/ saude_foco/artigos/ano2015/seguranca_paciente.pdf.

4. Reis CT, Laguardia J, Martins M. Adaptação transcultural da versão brasileira do Hospital Survey on Patient Safety Culture: etapa inicial. Cad. Saúde Pública. [Internet] 2012;28(11) [acesso em 12 fev 2016]. Disponível: http:// dx.doi.org/10.1590/S0102-311X2012001100019.

5. Sorra JS, Nieva VF. Hospital Survey on Patient Safety Culture. Rockville, MD: Agency for Healthcare Research and Quality; 2004.

6. Mardon RE, Khanna K, Sorra J, Dyer N, Famolaro T. Exploring relationships between hospital patient safety culture and adverse events. J Patient Saf. [Internet] 2010;6(4) [acesso em 10 set 2016]. Disponível: https://doi. org/10.1097/PTS.0b013e3181fd1a00.

7. Santiago THR, Turrini RNT. Cultura e clima organizacional para segurança do paciente em Unidades de Terapia Intensiva. Rev. esc. enferm. USP. [Internet] 2015;49(n.esp) [acesso em 05 jul 2016]. Disponível: http://dx.doi. org/10.1590/S0080-623420150000700018.

8. Agency for Healthcare Research and Quality (AHRQ). Hospital Survey on Patient Safety Culture. Contentlastupdated. [Internet] 2016 [acesso em 21 jul 2016]. Disponível: https://www.ahrq.gov/sites/default/files/ wysiwyg/professionals/quality-patient-safety/patientsafetyculture/hospital/2016/2016_hospitalsops_report_pt1. 
pdf9.

9. Tomazoni A, Rocha PK, Kusahara DM, de Souza AIJ, Macedo TR. Evaluation of the patient safety culture in neonatal intensive care. Texto Contexto Enferm. [Internet] 2015;24(1) [acesso em 20 jan 2017]. Disponível: http:// dx.doi.org/10.1590/0104-07072015000490014.

10. Claro CM, Krocockz DVC, Toffolleto MC, Padilha KG. Eventos adversos em Unidade de Terapia sobre a cultura não punitiva. Rev esc. enferm. USP. [Internet] 2011;45(1) [acesso em 20 jan 2017]. Disponível: http://dx.doi. org/10.1590/S0080-62342011000100023.

11. Bião e Silva ACA, Santa Rosa DO. Patient safety culture in hospital organization. Cogitare Enferm. [Internet] 2016;21(5) [acesso em 10 fev 2017]. Disponível: http://dx.doi.org/10.5380/ce.v21i5.45583.

12. de Souza VS, Kawamoto AM, de Oliveira JLC, Tonini NS, Fernandes LM, Nicola AL. Errors and adverse events: the interface with health professionals' safety culture. Cogitare Enferm. [Internet] 2015;20(3) [acesso em $12 \mathrm{fev}$ 2017]. Disponível: http://dx.doi.org/10.5380/ce.v20i3.40687.

13. Cauduro FLF, Sarquis LM, Sarquis LMM, Cruz EDA. Safety culture among surgical center professionals. Cogitare Enferm. [Internet] 2015;20(1) [acesso em 30 jan 2017]. Disponível: http://dx.doi.org/10.5380/ce.v20i1.36645.

14. Matiello RDC, Lima EFA, Coelho MCR, Oliveira ERA, Leite FMC, Primo CC. Patient safety culture from the perspective of nurses. Cogitare Enferm. [Internet] 2016;21(5) [acesso em 12 fev 2017]. Disponível: http://dx.doi. org/10.5380/ce.v21i5.45408.

15. Novaretti MCZ, Santos EV, Quitério LM, Daud-Gallotti RM. Nursing workload and occurrence of incidents and adverse events in ICU patients. Rev. bras. enferm. [Internet] 2014;67(5) [acesso em 05 jul 2016]. Disponível: http://dx.doi.org/10.1590/0034-7167.2014670504.

16. da Silva-Batalha EMS, Melleiro MM. Cultura de segurança do paciente em um hospital de ensino: diferenças de percepção existentes nos diferentes cenários dessa instituição. Texto Contexto Enferm. [Internet] 2015;24(2) [acesso em 19 out 2016]. Disponível: http://dx.doi.org/10.1590/0104-07072015000192014.

17. Minuzz AP, Salum NC, Locks MOH. Avaliação da cultura de segurança do paciente em terapia intensiva na perspectiva da equipe de saúde. Texto Contexto Enferm. [Internet] 2016;25(2) [acesso em 19 out 2016]. Disponível: http://dx.doi.org/10.1590/0104-07072016001610015.

18. Frota MA, Rolim KMC. Aspectos Gerais da formação da enfermagem: O perfil da formação dos enfermeiros, técnicos e auxiliares de enfermagem - Debatedor 1. Enferm. Foco. [Internet] 2016;(7) [acesso em 21 jan 2016]. Disponível: https://doi.org/10.21675/2357-707X.2016.v7.nESP.688.

19. Brasil. Ministério da Saúde. Portaria $n^{\circ} 529$, de $1^{\circ}$ de abril de 2013. Institui o Programa Nacional de Segurança do Paciente (PNSP). [Internet] Diário Oficial da União, 02 abr 2013 [acesso em 27 ago 2016]. Disponível: http:// bvsms.saude.gov.br/bvs/saudelegis/gm/2013/prt0529_01_04_2013.html.

20. Kawamoto AM, de Oliveira JLC, Tonini NS, Nicola AL. Liderança e cultura de segurança do paciente: percepções de profissionais em um hospital universitário. J. res.: fundam. care. online. [Internet] 2016;8(2) [acesso em 05 jul 2016]. Disponível: http://www.seer.unirio.br/index.php/cuidadofundamental/article/view/4530/pdf_1888.

21. Verbeek-Van Noord IN, Wagner C, Van-Dyck C, Twisk JWR, Bruijne MC. Is culture associated with patient safety in the emergency department? A study of staff perspectives. Int J Qual Health Care. [Internet] 2014;26(1) [acesso em 18 jul 2016]. Disponível: http://dx.doi.org/10.1093/intqhc/mzt087.

22. El-Jardali F, Dimassi H, Jamal D, Jaadar M, Hemadeh N. Predictors and outcomes of patient safety culture in hospitals. BMC Health Serv Res. [Internet] 2011;11(45) [acesso em 17 jul 2016]. Disponível: http://dx.doi. org/10.1186/1472-6963-11-45.

23. Wang X, Liu K, You LM, Xiang JG, Hu HG, Zhang LF, et al. The relationship between patient safety culture and adverse events: a questionnaire survey. Int J Nurs Stud. [Internet] 2014;51(8) [acesso em 10 set 2016]. Disponível: https://doi.org/10.1016/j.ijnurstu.2013.12.007.

24. Weaver SJ, Lubomski LH, Wilson RF, Pfoh ER, Martinez KA, Dy SM. Promoting a culture of safety as a patient safety strategy:a systematic review. Ann Inter Med. [Internet] 2013;158(5) [acesso em 10 set 2016]. Disponível: http://dx.doi.org/10.7326/0003-4819-158-5-201303051-00002. 